\title{
Optical and structural investigation of SiGe/Si quantum wells
}

\author{
L. Vescan, A. Hartmann, K. Schmidt, Ch. Dieker, and H. Lüth \\ Institute for Thin Films and Ion Technology, Research Center Jülich, 5170-Jülich, Germany \\ W. Jäger \\ Institute for Solid State Physics, Research Center Jülich, 5170-Jülich, Germany
}

(Received 27 November 1991; accepted for publication 26 February 1992)

\begin{abstract}
In this letter we report photoluminescence and structural results obtained on asymmetrically strained $\mathrm{Si}_{0.7} \mathrm{Ge}_{0.3} / \mathrm{Si}$ single and multiple quantum wells epitaxially grown by low pressure chemical vapor deposition. Well-resolved peaks were obtained which can be attributed to quantum well excitons and their transversal optical phonon replica. A good correlation between peak properties and structure results was found. From the photoluminescence peak energies a valence band offset of $0.27 \mathrm{eV}$ and an effective hole mass of 0.25 were estimated.
\end{abstract}

Photoluminescence (PL) is a powerful method widely used in later years to study the electronic states in quantum wells. For $\mathrm{SiGe} / \mathrm{Si}$ heterostructures there have been several reports of PL for various samples from unstrained bulk to atomic layer superlattices. For unstrained bulk $\mathrm{Si}_{1-x} \mathrm{Ge}_{x}$ alloys Weber and Alonso ${ }^{1}$ presented a detailed study of the near-band-gap PL. Sharp lines were detected in unstrained $\mathrm{Si}_{1-x} \mathrm{Ge}_{x}$ alloys grown by liquid phase epitaxy. ${ }^{2}$ Broad PL peaks were detected on molecular beam epitaxy (MBE) grown single layers. ${ }^{3,4}$ Intense PL was reported by Noël et $a l .{ }^{4}$ for MBE-grown single (SQW) and multiple quantum wells (MQW). There are several reports on strained $\mathrm{Si}_{m^{-}}$ $\mathrm{Ge}_{n}$ superlattices grown by $\mathrm{MBE}^{5-7}$ In particular, it has been predicted that for certain values of $m$ and $n$ and specific strain conditions, direct or quasi-direct band-gap material should be obtained in these superlattices. ${ }^{8}$

In the present study, $\mathrm{SiGe} / \mathrm{Si} \mathrm{SQW}$ and MQWs were epitaxially grown by low pressure chemical vapor deposition (LPCVD) and characterized by PL, Rutherford backscattering (RBS), and transmission electron microscopy (TEM). We observe several well-defined PL lines at low temperatures, with linewidths of $\sim 7-30 \mathrm{meV}$ depending on quantum well (QW) thickness. The comparison between measured and calculated PL energies allowed us to estimate the valence-band offset $\left(\Delta E_{V}\right)$ and the effective mass for holes $\left(m_{h}^{*}\right)$.

The QW structures were deposited on $p$-type $\mathrm{Si}(100)$ wafers, $2000 \Omega \mathrm{cm}$. The epitaxy was carried out at $700^{\circ} \mathrm{C}$ and 0.1 Torr in a LPCVD system ${ }^{9}$ using as source gases dichlorosilane and germane. Usual growth rates were for $\mathrm{Si}$ $2 \mathrm{~nm} / \mathrm{min}$ and for $\mathrm{Si}_{0.7} \mathrm{Ge}_{0.3} 1.8 \mathrm{~nm} / \mathrm{min} .^{10}$

The following layer sequences were chosen: for the MQWs: $\sim 260 \mathrm{~nm} \mathrm{Si} \mathrm{S}_{0.93} \mathrm{Ge}_{0.07}$ buffer and then the MQWs consisting of $\mathrm{Si}_{0.7} \mathrm{Ge}_{0.3}$ wells and $\mathrm{Si}$ barriers (see Table I) and for SQWs: $\sim 1 \mu \mathrm{m} \mathrm{Si} \mathrm{layer,} \mathrm{a} \mathrm{Si}_{0.7} \mathrm{Ge}_{0.3} \mathrm{SQW}$ layer, and a $\mathrm{Si}_{0.98} \mathrm{Ge}_{0.02}$ cap layer (see Table II).

The thickness of the QWs was determined by RBS using $1.4 \mathrm{MeV}^{4} \mathrm{He}^{+}$ions, tilting the sample $81^{\circ}$ to the $\mathrm{He}^{+}$ beam and fitting the measured data with calculated ones using the program RUMP. ${ }^{11}$ TEM characterization of the SQW and MQW structures was performed with a JEOL $4000 \mathrm{FX}$ electron microscope at $400 \mathrm{kV}$ on plan-view and cross-section specimens. The PL was measured at $4.2 \mathrm{~K}$ using a Fourier transform spectrometer with a $\mathrm{Ge} p-i-n$ diode cooled with liquid nitrogen as a detector. The samples were optically excited by $\mathrm{Ar}^{+}$laser light with a power density of $\sim 10 \mathrm{~W} / \mathrm{cm}^{2}$.

In Fig. 1 typical PL spectra from $\mathrm{Si}_{0.7} \mathrm{Ge}_{0.3} / \mathrm{Si} \mathrm{MQWs}$ of various well thicknesses are presented, together with the PL spectrum of the Si substrate itself. Three main features are observed in the PL spectra of the MQWs. We group them into lines labeled from $a$ to $d$, lines $D$, and lines from the substrate. Visible in the pure-substrate PL spectrum is a complex of exciton lines. This consists of free exciton lines (FE) with phonon participation (TO, LO, TA, TO $\left.+\mathrm{O}_{\Gamma}\right)$ and bound excitons without phonon and its phonon replica. ${ }^{12}$ The broad feature labeled EHD is duc to the electron-hole-droplet. All these substrate lines were observed, with one exception, in the MQW spectra, too, but reduced in intensity. The exception is the EHD line, which is absent in all MQWs.

First, we will discuss lines $a$ to $d$ (see also Table I). Line $d$ is well resolved in the $1 \mathrm{~nm} \mathrm{MQW}$, in the $1.2 \mathrm{~nm}$ sample it lies in the region of substrate lines, in the 1.5 and $2 \mathrm{~nm}$ samples it is again well defined. The line $c$ overlaps the substrate lines in the $1 \mathrm{~nm}$ sample, but for thicker wells it is again well separated from the substrate lines. The dominant characteristic of all four lines $a$ to $d$ is their shift to lower energy as the well thickness increases, which undoubtedly can be correlated with transitions of electrons from the condition band to QW-confined holes. The linewidth of lines $a$ to $d$ is for MQWs up to $1.5 \mathrm{~nm}$ relatively small (7-10 meV), while broadening occurs for the $2 \mathrm{~nm}$ MQW. Another interesting characteristic is that lines $a$ and $c$ seem to be correlated. Their energy difference is $\sim 57$

TABLE I. Data for MQW samples: thickness $d$ (in nm) and PL peaks. For all MQWs: buffer $x=0.07, d=260 \mathrm{~nm}$; QW: $x=0.3 ; n$ is the number of periods.

\begin{tabular}{|c|c|c|c|c|c|c|c|c|}
\hline \multicolumn{3}{|c|}{ MQW } & \multicolumn{6}{|c|}{ PL lines ( $\mathrm{meV}$ ) } \\
\hline$d_{\text {SIGe }}$ & $d_{\mathrm{Si}}$ & $n$ & $a$ & $b$ & $c$ & $d$ & $d-b$ & $c-a$ \\
\hline 1.0 & 12 & 10 & 1031 & 1051 & 1089 & 1109 & 58 & 58 \\
\hline 1.0 & 12 & 20 & 1031 & 1050 & 1089 & 1109 & 58 & 58 \\
\hline 1.2 & 14 & 20 & 1021 & 1034 & 1078 & 1093 & 59 & 57 \\
\hline 1.5 & 13 & 20 & 1008 & 1017 & 1064 & 1075 & 58 & 56 \\
\hline 2.0 & 14 & 20 & & 984 & 1026 & 1041 & 57 & \\
\hline
\end{tabular}


TABLE II. Data for SQW samples: thickness $d$ and energies of PL lines; cap layer: $x=0.02$; SQW: $x=0.3$.

\begin{tabular}{ccccccc}
\hline \hline \multirow{2}{*}{$\begin{array}{c}\text { Cap layer } \\
d / \mathrm{nm}\end{array}$} & SQW & \multicolumn{5}{c}{ PL lines/meV } \\
& $d / \mathrm{nm}$ & $a$ & $b$ & $c$ & $d$ & $d-b$ \\
\hline 360 & 2.2 & & 977 & 1018 & 1034 & 57 \\
500 & 2.6 & & 912 & & 963 & 51 \\
580 & 2.7 & & 894 & & 947 & 53 \\
330 & 3.5 & & 888 & & 938 & 50 \\
17 & 5.0 & & 865 & & 920 & 55 \\
13 & 5.8 & & 959 & & 914 & 55 \\
\hline \hline
\end{tabular}

$\mathrm{meV}$, while the same is valid for the line pair $b$ and $d$ having an energy difference of $\sim 58 \mathrm{meV}$.

The lines $D_{1}$ and $D_{2}$ do not shift when the well thicknesses increase, but they become more intense for thicker wells. Their energetic position coincides with the dislocation lines $D_{1}(0.812 \mathrm{eV})$ and $D_{2}(0.875 \mathrm{eV})$ identified in deformed silicon ${ }^{13}$ and were also observed in relaxed SiGc. $^{4,7}$

Figure 2(a) presents a MQW structure with wells of $1.5 \mathrm{~nm}$ thickness in a cross-section TEM micrograph showing a high degree of perfection characterized by layers of homogeneous thickness and abrupt and planar interfaces. This demonstrates, that this MQW structure is fully strained. A network of misfit dislocations has been identified at the buffer-substrate interface, visible as localized strained regions, which are undoubtedly related to the $D_{1}$ and $D_{2}$ lines in the PL spectrum of this sample. Figure 2(b) shows a cross-section TEM micrograph of a $2 \mathrm{~nm}$ MQW structure displaying regions of high structural perfection and regions within the individual wells with a continuously changing well thickness and/or composition, resulting in increasing bulging of the multilayers in growth direction. Misfit dislocations are present only at the buffer-

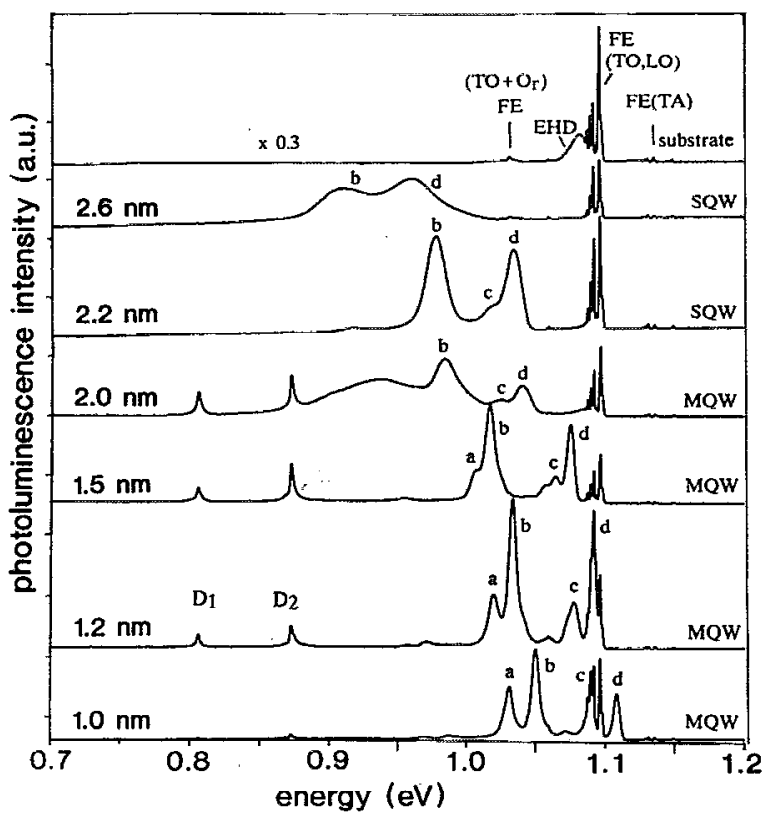

FIG. 1. PL spectra of $\mathrm{Si}_{0.7} \mathrm{Ge}_{0.3} / \mathrm{Si} \mathrm{MQW}$ and SQW structures with different well thicknesses and of the substrate alone, measured at $T \sim 4.2 \mathrm{~K}$.
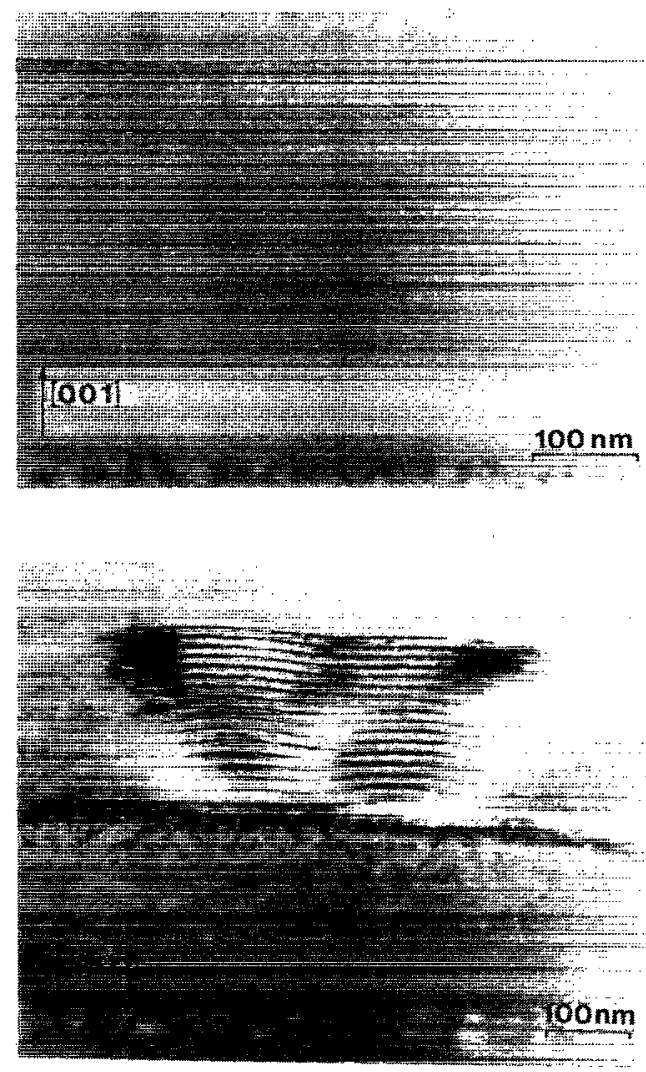

FIG. 2. Cross-section TEM micrograph of 20-period MQW structures grown on $\mathrm{Si}_{0.93} \mathrm{Ge}_{0.07}$ buffers; bright field images were taken with electron beam parallel to interfaces near a $\langle 110\rangle$ projection. The corresponding $P L$ are shown in Fig. 1. Nominal well width: (a) $1.5 \mathrm{~nm}$ and (b) $2 \mathrm{~nm}$.

substrate interface, but not at all at the well interfaces and no threading dislocations were observed. The formation of such regions (lateral extension $\sim 100-500 \mathrm{~nm}$ ) was observed for all samples with nominal thickness of the SiGe wells $>2 \mathrm{~nm}$. It also occurred in SQWs, if this layer thickness is exceeded.

The SQWs investigated have a nominal well thickness of 2.2 to $6 \mathrm{~nm}$ (see Table II). In Fig. 1 PL spectra of two SQW structures are shown. Similar to the MQWs, the QW-related lines $b$ to $d$ of the SQW samples shift to lower energy as the well becomes thicker, and for wells $>2.5 \mathrm{~nm}$ only lines $d$ and $b$ are detected, with an energy difference of $\sim 55 \mathrm{meV}$. No dislocation lines were detected for all SQWs investigated (see Table II). Similar to the MQWs, inhomogeneous regions are also observed by TEM for all SQWs investigated, but no misfit dislocations at the interfaces and no threading dislocations are detected which explains the absence of the $D_{1}$ and $D_{2}$ lines in the PL spectra of the SQWs. We can conclude, that for the SQW and MQW structures based on asymmetrically strained $\mathrm{Si}_{0.7} \mathrm{Ge}_{0.3} / \mathrm{Si}$ the inhomogeneities in the SiGe layers were obviously the cause for the broadening of the PL lines $b$ and $d$ and the disappearance of lines $a$ and $c$.

We start the discussion with the PL lines $a$ and $b$. Tables I and II show, that these lines lie 50-58 meV below the $c$ and $d$ lines. In bulk $\mathrm{Si}_{1-x} \mathrm{Ge}_{x}$ alloys Weber and Alonso ${ }^{1}$ identified the $\mathrm{TO}_{\mathrm{Si}-\mathrm{Si}}$ phonon with $58 \mathrm{meV}$ and the 


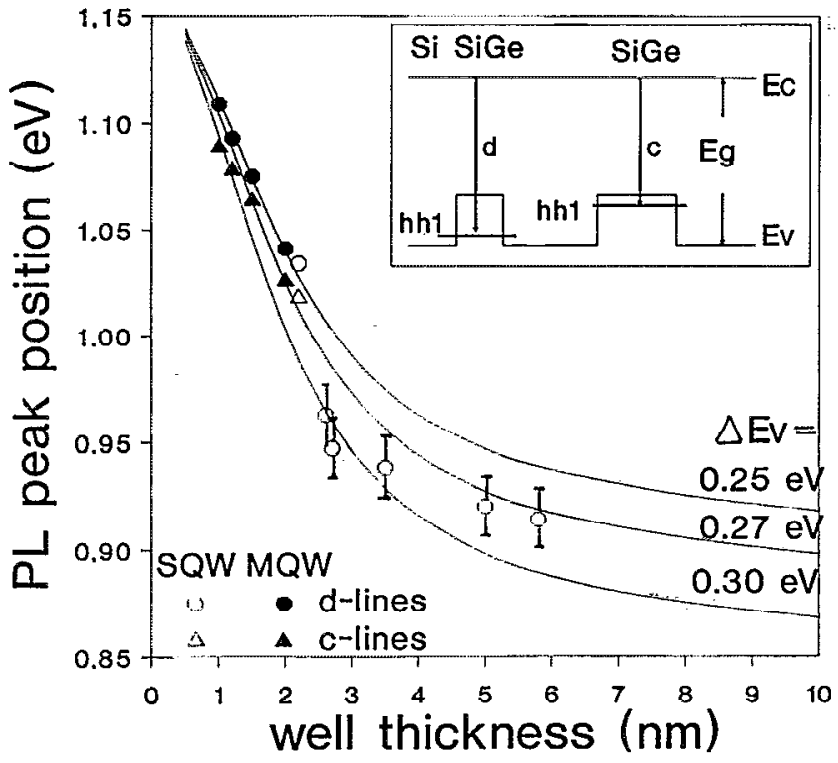

FIG. 3. Calculated and experimental PL peaks as a function of the well thickness. Only no-phonon QW exciton lines $c$ and $d$ are represented. (a) , $\triangle \mathrm{MQW}$, (b) $\mathrm{O}, \triangle \mathrm{SQW}$.

$\mathrm{TO}_{\mathrm{Si}-\mathrm{Ge}}$ with $49 \mathrm{meV}$. This allows us to interpret lines $a$ and $b$ as TO replicas of the PL lines $c$ and $d$, respectively. The $c$ and $d$ lines we assign to no-phonon transitions due to QW excitons, splitted into a doublet. Doublet splitting in the photoluminescence spectra of III-V heterostructures was explained as being due to 1 monolayer thickness variation if the lateral extension of the thickness fluctuations is greater than the Bohr radius of the confined excitons. ${ }^{14}$ In our case, the QW exciton lines $c$ and $d$ lie $\sim 20 \mathrm{meV}$ apart, which is equivalent to $\sim 2$ monolayers thickness variation. This was observed for well thicknesses $<2 \mathrm{~nm}$.

In order to analyze the no-phonon QW-exciton lines more quantitatively, we calculate the quantized state energies $E_{i}$ in the QW using different values for $m_{h}^{*}$ in the well and the barrier. No dependence on strain of $m_{h}^{*}$ was taken into account. The energetic position of the PL lines is equal to $E_{g}-\Delta E_{V}+E_{i}$ and is represented in Fig. 3 as a function of the well thickness for $i=1$. Here $E_{g}$ is the gap for cubic Si. Also represented are the QW-exciton lines $c$ and $d$. A reasonable fit was obtained with $m_{h}^{*}=0.25 \mathrm{~m}$ and $\Delta E_{V}$ $=0.27 \mathrm{eV}$. The disappearance of splitting for wells $>2.5$ $\mathrm{nm}$ and broadening of the QW-exciton line $d$ is due to an increase of thickness fluctuations, which is in agreement with the TEM results.

In conclusion, the photoluminescence of asymmetrically strained $\mathrm{Si}_{0.7} \mathrm{Ge}_{0.3} / \mathrm{Si} \mathrm{MQW}$ and SQW structures is due to $\mathrm{QW}$ excitons and their TO replicas indicating the indirect character of this system. It was possible to estimate a valence band offset of $0.27 \mathrm{eV}$ and an effective hole mass of $0.25 \mathrm{~m}$. Splitting of the no-phonon QW-exciton lines is very probably due to thickness fluctuations. In addition, we found that the PL dislocation lines $D_{1}$ and $D_{2}$ are well correlated with misfit dislocations at the buffer substrate interface.

It is a pleasure to acknowledge helpful conversations with R. Carius, K. Wolter, H. P. Tang, and S. Mantl and the technical assistance of $\mathrm{K}$. Wambach.

${ }^{1}$ J. Weber and M. I. Alonso, Phys. Rev. B. 40, 5683 (1989).

${ }^{2}$ P. O. Hansson, J. H. Werner, L. Tapfer, L. P. Tilly, and E. Bauser, J. Appl. Phys. 68, 2158 (1990).

${ }^{3}$ V. Higgs, G. Davies, and R. Kubiak, Mater. Sci. Forum 65-66, 351 (1990).

${ }^{4}$ J.-P. Noël, N. L. Rowell, D. C. Houghton, and D. D. Popovic, Appl. Phys. Lett. 57, 1037 (1990).

${ }^{5}$ G. Abstreiter, K. Eber1, E. Fries, W. Wegschneider, and R. Zachai, J. Cryst. Growth 95, 431 (1990).

${ }^{6}$ E. A. Montie, G. F. A. van der Walle, D. J. Gravesteijn, and A. A. van Gorkum, Appl. Phys. Lett. 56, 340 (1990).

${ }^{7}$ K. Miki, H. Okumura, K. Sakamoto, H. Matsuhata, T. Sakamoto, and S. Yoshida, J. Cryst. Growth 111, 340 (1991).

${ }^{8}$ S. Froyen, D. M. Wood, and A. Zunger, Phys. Rev. B 37, 6893 (1988).

${ }^{9}$ L. Vescan, H. Beneking, and O. Meyer, J. Cryst. Growth 76, 63 (1986).

${ }^{10}$ H. P. Tang, L. Vescan, and H. Lüth, J. Cryst. Growth 116, 1 (1992).

${ }^{11}$ L. R. Doolittle, Nucl. Instrum. Methods B 9, 344 (1985).

${ }^{12}$ Properties of Silicon, EMIS Datareviews Series No. 4 (INSPEC, London, 1988), pp. 209-213.

${ }^{13}$ R. Sauer, J. Weber, J. Stolz, E. R. Weber, K.H.-Küsters, and H. Alexander, Appl. Phys. A 36, 1 (1985).

${ }^{14}$ J. Christen, Festkörperprobleme 30, 239 (1990). 